\title{
Live births from isolated primary/early secondary follicles following a multistep culture without organ culture in mice
}

\author{
Nahoko Mochida ${ }^{1}$, Akiko Akatani-Hasegawa ${ }^{1,2}$, Kayo Saka ${ }^{1}$, Mai Ogino ${ }^{1}$, Yoko Hosoda ${ }^{1}$, \\ Ryu Wada ${ }^{1}$, Hideaki Sawai ${ }^{1}$ and Hiroaki Shibahara ${ }^{1}$ \\ ${ }^{1}$ Department of Obstetrics and Gynecology, Hyogo College of Medicine, Mukogawa-cho 1-1, Nishinomiya, Hyogo \\ 663-8501, Japan and ${ }^{2}$ The Institute of Experimental Animal Sciences, Hyogo College of Medicine, Hyogo, Japan \\ Correspondence should be addressed to H Shibahara; Email: sibahara@hyo-med.ac.jp
}

\begin{abstract}
Although the ovary has a large store of germ cells, most of them do not reach mature stages. If a culture system could be developed from early growing follicles to mature oocytes, it would be useful for biological research as well as for reproductive medicine. This study was conducted to establish a multistep culture system from isolated early growing follicles to mature oocytes using a mouse model. Early growing follicles with diameters of 60-95 $\mu \mathrm{m}$ corresponding to primary and early secondary follicles were isolated from 6-day-old mice and classified into three groups by diameter. These follicles contained oocytes with diameters of $\sim 45 \mu \mathrm{m}$ and one or a few layered granulosa cells on the basal lamina. Embedding in collagen gel was followed by first-step culture. After 9-day culture, the growing follicles were transferred onto collagen-coated membrane in the second step. At day 17 of the culture series, the oocyte-granulosa cell complexes were subjected to in vitro maturation. Around $90 \%$ of the oocytes in follicles surviving at day 17 resumed second meiosis (metaphase II oocytes: 49.0-58.7\%), regardless of the size when the follicle culture started. To assess developmental competence to live birth, the eggs were used for IVF and implantation in pseudopregnant mice. We successfully obtained two live offspring that produced next generations after puberty. We thus conclude that the culture system reported here was able to induce the growth of small follicles and the resultant mature oocytes were able to develop into normal mice.
\end{abstract}

Reproduction (2013) 146 37-47

\section{Introduction}

In mammalian ovaries, there are numerous follicles at various stages of growth. Especially, gonadotropinindependent small follicles are abundant but most of them do not reach ovulation. If a whole culture system from small follicles to preovulatory follicles can be established, it would be useful for follicular biology and future infertility therapy. Such a culture system provides a valuable model to study critical interactions between oocyte and follicular cells and factors regulating follicle development at each developmental stage. Eventually, follicle culture techniques could be applied to fertility preservation.

Through advances in aggressive chemotherapy and/or radiotherapy as well as abdominal surgery, survival rates from cancers have significantly increased. However, such therapies also damage normal cells including gametes, resulting in cancer survivors becoming infertile due to ovarian failure (Meirow \& Nugent 2001, Lutchman Singh et al. 2005, Anderson et al. 2008). Women with cancer may cryopreserve their own mature eggs before starting aggressive cancer therapy, but the numbers that can be used for infertility therapy are very small. Ovarian tissue cryopreservation is an option to recover fertility. For prepubertal girls, ovarian tissue cryopreservation is the only way to preserve their future fertility. Recently, ovarian tissue autografting after cryopreservation has been applied to a young patient who had recovered from Hodgkin's lymphoma and it resulted in a healthy baby being born (Donnez et al. 2004). Subsequent successful cases have been reported (Meirow et al. 2005, Demeestere et al. 2007, Andersen et al. 2008). However, autografting carries a risk of reintroduction of malignant cells in patients who have recovered from cancer (Shaw et al. 1996, Meirow et al. 2008). The technologies of in vitro growth (IVG) of follicles or oocyte-granulosa cell complexes (OGCs), in vitro maturation (IVM) of oocytes, and IVF are feasible methods for such patients.

Many researchers have reported about IVG of immature oocytes in mice (Eppig \& Schroeder 1989, Nayudu \& Osborn 1992, Cortvrindt et al. 1996, Eppig \& O'Brien 1996, Lenie et al. 2004). Induction and maintenance of follicle growth are more difficult in earlier stages of preantral follicles, which require longer culture periods to reach mature stages (Smitz \& Cortvrindt 2002, Hirao \& Miyano 2008). It is well 
established that folliculogenesis and meiotic maturation of the oocyte are strictly timed processes. To reach fully grown stages, intrinsic culture periods are necessary depending on follicular diameters at the start of the culture. For recruitment of dormant primordial follicles in culture, a multistep culture system including organ culture is necessary before IVG of isolated OGCs or isolated follicles (Eppig \& O'Brien 1996, Telfer et al. 2008, Jin et al. 2010).

In the ovary, the recruited primary follicles start to grow in the preantral stages until antrum formation. Preantral folliculogenesis consists of several further stages as follows: i) oocytes and one-layered granulosa cells are present, ii) granulosa cell proliferation from one layer to two to three layers, iii) granulosa cell layers increase from a few layers to four to six layers, and iv) fully grown preantral follicles having seven to eight granulosa cell layers capable of forming an antrum. In this article, follicles at each stage of preantral folliculogenesis from the above-mentioned stages are designated as primary, early secondary, middle secondary, and late secondary follicles respectively. Primary/early secondary follicles are presumed to be gonadotropin independent and middle/late secondary follicles are considered to be gonadotropin dependent. Development of optimal culture systems for different follicle stages is necessary for application to reproductive technology because mammalian reproductive ovaries contain follicles at various growth stages. In animal experiments, middle/late secondary follicles have been reported to grow in culture achieving live births, while primary and early secondary follicles are still very difficult to be grown in culture. The immature granulosa cells at these stages do not organize normal follicle shape under culture conditions.

To keep the adequate follicle structure during culture, different approaches have been attempted. All of them stated that keeping three-dimensional structure of follicles was important for successful growth of follicles. First, polyvinylpyrrolidone (PVP) was applied to the culture medium. PVP gave viscosity to the culture medium and prevented dispersal of growth factors released by follicular cells (Hirao et al. 2004). Secondly, an inverted drop method was reported. This method prevents follicular cells from attachment and spread on the bottom of the culture dish (Wycherley et al. 2004). Thirdly, embedding follicles in biomaterial gels such as collagen (Torrance et al. 1989, Carroll et al. 1991), alginate (Pangas et al. 2003), and matrigels (Hwang et al. 2000, Scott et al. 2004) was adopted for the culture. More recently, alginate prepared from brown algae has been shown to give more successful results in various animal species including mice (Kreeger et al. 2006, Xu et al. 2006), monkeys (Xu et al. 2009b), and humans (Xu et al. 2009a).

Maintaining three-dimensional structure of follicles is important for keeping interactions between the cells themselves and the extracellular matrix to achieve mature stages. Cross-linked biomaterials should retain the growth factors around the oocyte and help the formation of local gap junctions between oocytes and granulosa cells. Naturally occurring extracellular matrices such as fibronectin, laminin, and collagen are deposited in ovarian follicles during follicle development. These matrices have important functions in a stage-specific manner (Berkholtz et al. 2006). In this study, we focused on collagen gel, because collagen has been reported to stimulate cell growth and development in various mammalian cell culture systems (Wicha et al. 1979, Yang et al. 1980, Yang \& Nandi 1983). Not only that, the protein is also found throughout the animal world and mainly constitutes connective tissues. It is believed to have a physical function such as maintaining morphology and strength of organs. Collagen is also an essential factor for ovarian folliculogenesis. The culture systems using collagen, therefore, may keep normal ovarian functions and support cell-cell communications, regulation of cell development, and biological signaling pathways from the extracellular environment.

In 1989, Eppig's group reported the use of collagencoated membrane for a culture method of OGCs (Eppig \& Schroeder 1989). This group also succeeded in obtaining live births from neonatal mouse ovary by organ culture followed by subsequent culture of OGCs that were isolated from 8-day-old ovaries chronologically (Eppig \& O'Brien 1996, O'Brien et al. 2003). Similarly, in our own research, preantral follicles isolated from cryopreserved ovaries of 16-day-old mice grew and reached mature stages in culture, and the oocytes were fertilized and resulted in live births (Hasegawa et al. 2006). However, primary/early secondary follicles having diameters of $<100 \mu \mathrm{m}$ with one to three layers of granulosa cells did not grow in these culture conditions. Oocyte developmental competence is defined as the oocyte's potential to undergo maturation, fertilization, development into blastocyst, and as a final outcome to give rise to live offspring. To our knowledge, live birth has not been achieved from such small follicles. This study was designed to establish an effective culture method for mouse primary/early secondary follicles with diameters of $<95 \mu \mathrm{m}$. For this purpose, we used 6-day-old mouse ovaries that do not contain middle secondary follicles with more than four layers of granulose cells. We also assessed the competence of the derived oocytes to achieve live births.

\section{Materials and methods}

\section{Animals and materials}

The mice used in this study were BDF1 females derived from matings between $\mathrm{DBA} / 2$ males and C57BL/6 females. ICR male mice (18-20 weeks old) and ICR pseudopregnant female mice (10-13 weeks old) were used for IVF and embryo transfer respectively. 
Animals were purchased (Japan SLC, Inc., Shizuoka, Japan; CLEA Japan, Inc., Tokyo, Japan) and housed in a temperature- and light-controlled environment on a $12 \mathrm{~h}$ light:12 $\mathrm{h}$ darkness photoperiod and were provided with food and water ad libitum. The animal experiments in this study were approved by the Committee on Animal Experimentation of Hyogo College of Medicine. Unless otherwise noted, all chemicals were purchased from Sigma-Aldrich.

\section{Follicle culture}

Late secondary follicles with diameters of 125-140 $\mu \mathrm{m}$ were collected from 16-day-old mice for IVG, as described in the previous report (Hasegawa et al. 2006). Primary/early secondary follicles surrounded by one or a few layers of somatic cells were mechanically dissected using $30 \mathrm{G}$ needles from 6-day-old BDF1 mouse ovaries. The follicles were isolated in L-15 medium (Invitrogen) supplemented with $20 \mathrm{mg} / \mathrm{ml}$ BSA and antibiotic antimycotic solution (penicillin, $10 \mathrm{IU} / \mathrm{ml}$; streptomycin, $10 \mu \mathrm{g} / \mathrm{ml}$; and amphotericin B, $25 \mathrm{ng} / \mathrm{ml})$. The follicles corresponding to classes $3 a$ and $3 b$ (Pedersen \& Peters 1968) were collected. The follicle classes were also confirmed by the number of granulosa cell layers in serial sections of whole ovaries stained with hematoxylin and eosin. We also calculated the number of granulosa cells per follicle. The isolated follicles were separated into oocytes and granulosa cells by treatments with collagenase and trypsin followed by repeated pipetting. Numbers of oocytes and granulosa cells were counted by a hematocytometer to determine the average number of granulosa cells surrounding an oocyte.

The collected follicles were divided into three groups based on the follicle diameters: group A, 80-95 $\mu \mathrm{m}$, two or three partial layers of granulosa cells; group B, $70-80 \mu \mathrm{m}$, granulosa cell layers similar to those in group A but diameters are smaller than those of group $A$; and group C, 60-70 $\mu \mathrm{m}$, one or two partial layers of granulosa cells. The isolated and grouped follicles were cultured in collagen gels for 9 days, which were designated IVGf-1. The follicles were transferred to a second culture with collagen-coated membrane for 8 days, which were designated IVGf- 2 . The details are as follows.

\section{In vitro growth of follicles-1}

Collagen gel (Cellmatrix; Nitta Gelatin, Inc., Osaka, Japan) was prepared according to the manufacturer's instructions. Briefly, $10 \mu \mathrm{l}$ of reconstituted collagen solution were poured into a $60 \mathrm{~mm}$ dish (FALCON 351007 Petri Dish: Becton Dickinson Labware, Franklin Lakes, NJ, USA) in the form of droplets as a base layer and allowed to gel for $30 \mathrm{~min}$ at $37^{\circ} \mathrm{C}$. Those base layers were used to prevent the follicles from attaching directly to the culture dish and growing there. The follicles in each group were washed three times in growth medium and ten follicles were put separately onto collagen gel base layer. Immediately after this, an additional collagen solution was poured onto the base layer to cover the follicles. Follicles were embedded in collagen gel. After the top layer was fixed, $100 \mu \mathrm{l}$ of growth medium were overlaid onto the follicle-containing gel to make microdroplets and then the microdroplets were covered with mineral oil. The medium used for IVGf-1 was $\alpha$-minimum essential medium (MEM) supplemented with $5 \%$ FCS, ITS (insulin, $10 \mu \mathrm{g} / \mathrm{ml}$; transferrin, $5.5 \mu \mathrm{g} / \mathrm{ml}$; and sodium selenite, $5 \mathrm{ng} / \mathrm{ml}$ ), antibiotic antimycotic solution (penicillin, $10 \mathrm{IU} / \mathrm{ml}$; streptomycin, $10 \mu \mathrm{g} / \mathrm{ml}$; and amphotericin B, $25 \mathrm{ng} / \mathrm{ml}), 1 \mathrm{mlU} / \mathrm{ml}$ of recombinant human FSH (Follistim: Organon, The Netherlands), and $1 \mathrm{ng} / \mathrm{ml}$ of mouse epidermal growth factor (mEGF). Follicles were cultured at $37^{\circ} \mathrm{C}$ in $5 \%$ $\mathrm{CO}_{2}, 5 \% \mathrm{O}_{2}$, and $90 \% \mathrm{~N}_{2}$ for 9 days. Half of the medium was changed every other day. On the first and ninth days of culture, follicle and zona pellucida diameter (excluding oocyte diameter) were determined by measuring two different axes (length and width) using an inverted microscope equipped with a micrometer. Oocyte-enclosing cell clusters having a diameter $>110 \mu \mathrm{m}$ were regarded as growing follicles.

\section{In vitro growth of follicles-2}

At day 9 of IVGf-1, growing follicles were removed from the collagen gel by treatment with $100 \mathrm{IU} / \mathrm{ml}$ collagenase (COLLAGENASE L: Nitta Gelatin, Inc.) for $20 \mathrm{~min}$ at $37^{\circ} \mathrm{C}$. Follicles were washed with IVGf-2 medium composed of $\alpha$-MEM, $5 \%$ FCS, ITS, antibiotic solution (penicillin, $10 \mathrm{IU} / \mathrm{ml}$; and streptomycin, $10 \mu \mathrm{g} / \mathrm{ml}$ ), and $100 \mathrm{mIU} / \mathrm{ml}$ of recombinant human FSH and transferred to the 12 -well plate $(2 \mathrm{ml} /$ well $)$ equipped with a collagen-coated membrane insert (Transwell-COL: Corning Incorporated Life Sciences, Tewksbury, MA, USA) and cultured in IVGf-2 medium for 8 days at $37^{\circ} \mathrm{C}$ in $5 \% \mathrm{CO}_{2}$ in air. Half of the medium was changed every other day. A follicle comprising an oocyte and granulosa cells attached to the collagen-coated membrane was considered to be a surviving follicle.

\section{IVM, IVF, and embryo transfer}

At day 17 of the whole culture series (IVGf-1 and IVGf-2), the surviving follicles were dislodged from the Transwell-COL membrane by pipetting and transferred to the maturation medium. As the maturation medium, IVGf-2 medium supplemented with $2.5 \mathrm{IU} / \mathrm{ml}$ hCG (Mochida Pharmaceutical Co., Ltd., Tokyo, Japan) and $10 \mathrm{ng} / \mathrm{ml} \mathrm{mEGF}$ was used. After $19 \mathrm{~h}$ of incubation, in vitro ovulation was observed. Most follicles released mucified COCs. Those COCs were collected to estimate the diameters of the oocytes and to assess oocyte nuclear maturation. The mature oocytes that underwent GVBD 

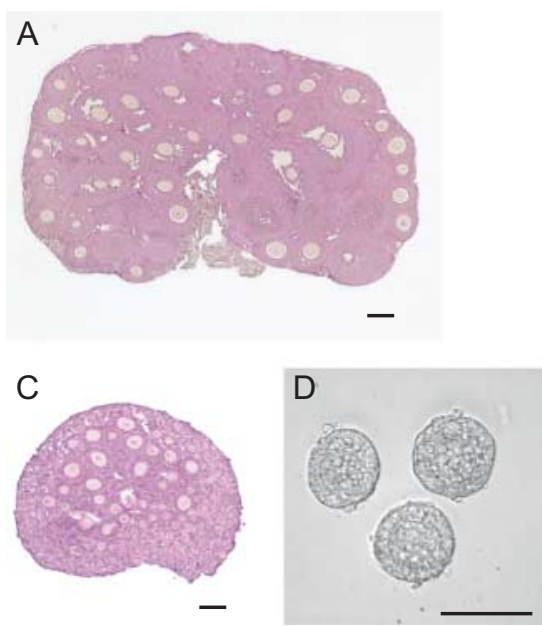
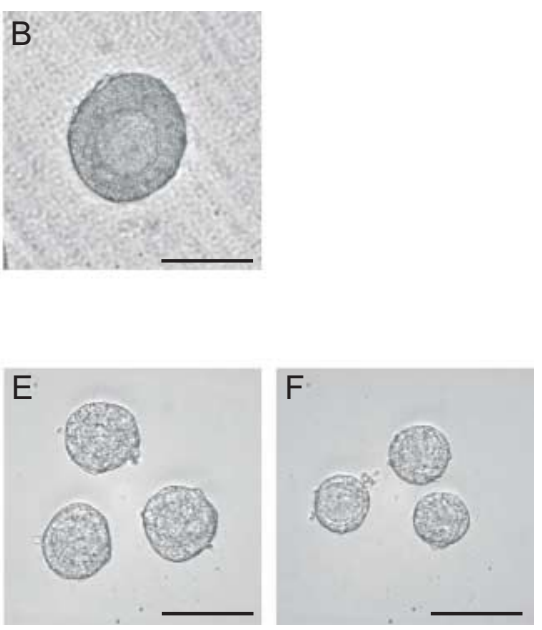

Figure 1 Comparison of 6 - and 16-day-old mouse ovaries and follicles. (A) Hematoxylin-eosin staining of 16-day-old mouse ovarian section. There are many preantral and early antral follicles. (B) A typical OGC derived from preantral follicles isolated from 16-day-old mouse ovaries. The follicles grew on collagen-coated membrane without collagen gel culture (IVGf-1).

(C) Hematoxylin-eosin staining of 6-day-old mouse ovarian section. There are many primordial follicles in the periphery region. Early secondary follicles are mostly found in the medullar region of the ovary. (D, E, and F) Follicles isolated from 6-day-old mouse ovaries were classified by their diameters. (D) Group A: follicle diameter is $80-95 \mu \mathrm{m}$. (E) Group B: follicle diameter is $70-80 \mu \mathrm{m}$. (F) Group C: follicle diameter is 60-70 $\mu \mathrm{m}$. Scale bar $=100 \mu \mathrm{m}$. or reached MII were transferred to modified HTF medium (zenith HTF for Mouse IVF: IVFonline.com, LLC, Guilford, CT, USA) for IVF. Sperm were collected from ICR mouse epididymis and used for insemination at $0.8 \times 10^{6} \mathrm{sperm} / \mathrm{ml}$. After $6 \mathrm{~h}$, oocytes were examined in fresh modified HTF medium. Fertilized zygote, MII stage oocyte, and GVBD oocyte were assessed by the presence of two pronuclei, by the extruded first polar body, and by no GV membrane respectively. They were further cultured overnight in the same medium and the resultant two-cell-stage embryos were cultured in modified KSOM (KSOMaa Evolve: IVFonline.com, LLC) for $96 \mathrm{~h}$ to examine their competence for development into blastocysts.

In the experiment for obtaining live offspring, two-cellstage embryos were vitrified by a minimum volume cooling method to collect sufficient number of embryos. Vitrification and warming were performed using vitrification/thawing kits (VT101; VT102: KITAZATO Co., Ltd., Shizuoka, Japan). Surviving embryos after warming were cultured in modified KSOM (KSOMaa Evolve: IVFonline.com, LLC) for $15 \mathrm{~h}$ and developing embryos at the four-cell stage were transferred into the oviducts of pseudopregnant ICR female mice (0.5 days postcoitum). Five to ten embryos were transferred to each uterine horn in a minimal volume of culture medium. Cesarean section was performed to deliver live offspring at 19.5 days postcoitum.

\section{Statistical analysis}

The results of follicle culture and embryo development were shown as mean percentages of multiple independent experiments. Differences among the three classified groups were examined using contingency tables and the $\chi^{2}$ test. One-way ANOVA followed by Tukey's multiple comparison test was used for statistical analysis of follicle and oocyte diameter. Differences were considered to be significant at $P<0.05$.

\section{Results \\ IVG of early secondary follicles compared with preantral follicles}

Preantral and early secondary follicles were collected from 16- and 6-day-old mouse ovaries respectively (Fig. 1A and C). The preantral follicles were covered with five to six layers of granulosa cells and their diameters were 125-140 $\mu$ m (Fig. 1B), while the early secondary follicles were covered with two to three layers of granulosa cells and their diameters were $<100 \mu \mathrm{m}$ (Fig. 1D). $95.5 \%$ of the preantral follicles grew in collagen-coated membrane culture after 6 days and the grown follicles were matured in IVM (Table 1). The resultant mature oocytes were then fertilized and the presumed embryos were cleaved. However, the early secondary follicles did not grow under these culture conditions (Table 1).

Table 1 Comparison of follicle growth between preantral stage and early secondary stage by collagen-coated membrane culture system.

\begin{tabular}{|c|c|c|c|c|c|}
\hline & Used follicles & $\begin{array}{c}\text { Grown follicles after } \\
6 \text { days }\end{array}$ & $\begin{array}{l}\text { Mature oocytes } \\
\text { (metaphase II) } \\
\text { after IVM }\end{array}$ & $\begin{array}{c}\text { Fertilized eggs } \\
(2 \mathrm{PN})\end{array}$ & Cleaved embryos \\
\hline $\begin{array}{l}\text { Preantral follicles } \\
\quad(125-140 \mu \mathrm{m})\end{array}$ & 134 & $128 / 134(95.5 \%)$ & $80 / 134(59.7 \%)$ & $47 / 80(58.8 \%)$ & $42 / 47$ (89.4\%) \\
\hline $\begin{array}{l}\text { Early secondary } \\
\quad \text { follicles }(<100 \mu \mathrm{m})\end{array}$ & 67 & $0 / 67(0 \%)$ & NA & NA & NA \\
\hline
\end{tabular}

PN, pronuclei; NA, not applicable. 


\section{IVG of primary and early secondary follicles collected from 6-day-old mouse ovaries}

To establish a novel culture system for primary/early secondary follicles, collagen gel culture (IVG of follicles-1 (IVGf-1)) was adopted before collagen-coated membrane culture (IVG of follicles-2 (IVGf-2)). The procedure is illustrated in Fig. 2. Follicles were isolated from 6-day-old mouse ovaries and classified into three groups based on the diameters (Fig. 1D, E, and F). In preliminary experiments of IVGf-1, we checked gas conditions and found that growth rates of follicles in $5 \% \mathrm{CO}_{2}, 5 \% \mathrm{O}_{2}$, and $90 \% \mathrm{~N}_{2}$ were better than those in $5 \% \mathrm{CO}_{2}$ in air. Under a condition of $5 \% \mathrm{CO}_{2}$ in air,
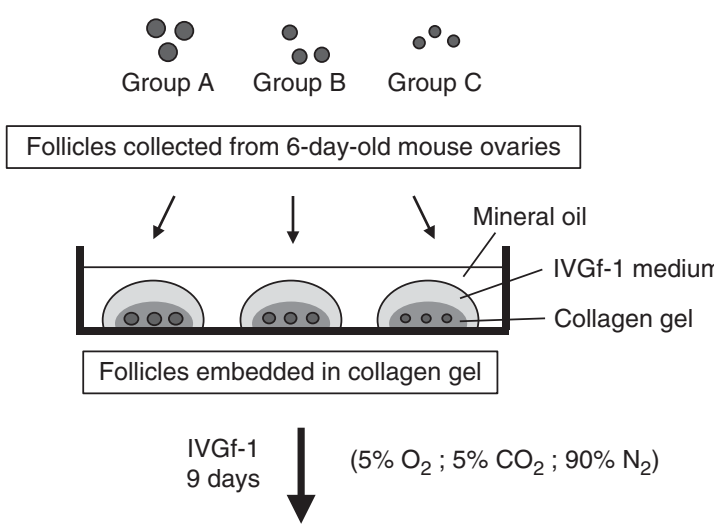

Collagenase treatment for follicle isolation
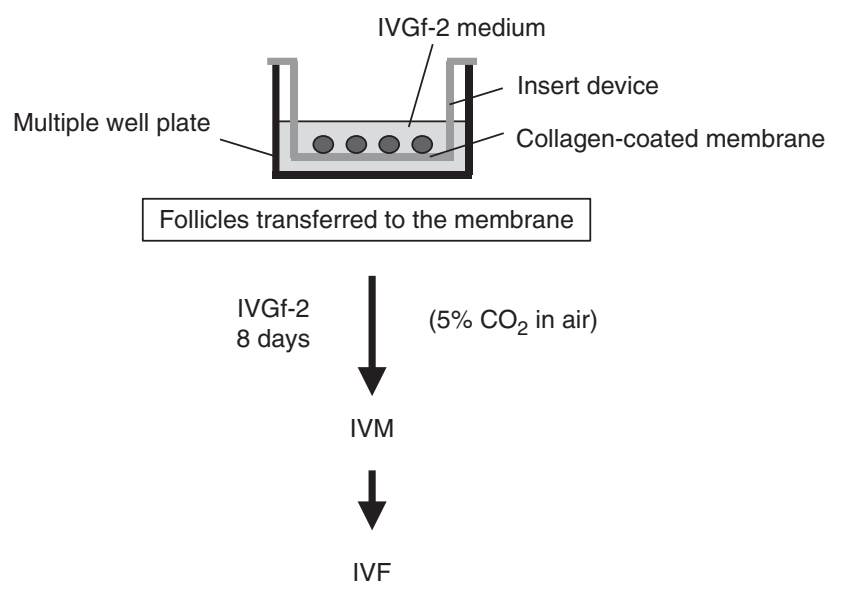

Figure 2 Schematic illustrating the two-step culture method. Intact follicles were mechanically isolated from 6-day-old mouse ovaries and classified into three groups, groups $\mathrm{A}, \mathrm{B}$, and $\mathrm{C}$, based on their diameters. Group A: $80-95 \mu \mathrm{m}$, group B: $70-80 \mu \mathrm{m}$, and group C: 60-70 $\mu \mathrm{m}$. Ten follicles in each group were embedded in a collagen gel microdroplet and cultured for 9 days (IVGf-1). Growing follicles were isolated by collagenase treatment to transfer to the collagen-coated membrane for a further culture (IVGf-2). About 30 follicles were cultured in a membrane insert. Medium components of IVGf-1 and IVGf-2 are described in the Materials and methods section. After 8 days, the resultant grown follicles were examined for maturation potential by in vitro maturation (IVM). Nuclear maturation and fertilizing ability of the oocyte were analyzed by IVF. some follicles from group A grew $25.0 \%$ (4/16) but none grew from group B or group $\mathrm{C}, 0 \%(0 / 16)$ or $0 \%(0 / 22)$ respectively. However, under $5 \% \mathrm{CO}_{2}, 5 \% \mathrm{O}_{2}$, and $90 \%$ $\mathrm{N}_{2}$ conditions, the follicles from groups $\mathrm{A}, \mathrm{B}$, and $\mathrm{C}$ grew at $71.4 \%(15 / 21), 52.2 \%(12 / 23)$, and $20.0 \%(4 / 20)$ respectively. Therefore, we chose the gas conditions of $5 \% \mathrm{CO}_{2}, 5 \% \mathrm{O}_{2}$, and $90 \% \mathrm{~N}_{2}$. After 9-day culture of IVGf- 1 , the follicles in groups $\mathrm{A}, \mathrm{B}$, and $\mathrm{C}$ were successfully growing at $78.7,48.6$, and $18.0 \%$ respectively (Table 2). Larger follicle groups resulted in higher growth rates. When the follicles isolated from the gel were transferred to IVGf- 2 and cultured for 8 days, they grew at $61.6,33.5$, and $9.9 \%$ in each group. The growth rates were also correlated with the follicle size at the beginning of culture. The oocytes in the fully grown follicles became fertilizable mature eggs at germinal vesicle breakdown (GVBD) or metaphase II (MII) stage after IVM culture. Typical morphology is shown in Fig. 3. Figure $3 \mathrm{~A}$ shows a collected early secondary follicle embedded in the collagen gel at day 0 of the culture. After 5-day culture of IVGf-1, the follicle was growing, which could be recognized by increased oocyte diameter and significantly thicker granulosa cell layers than at day 0 (Fig. 3B). Fibrous cells located around the edge region of the follicle spread outward. Zona pellucida was clearly observed as shown by the arrow. By day 9 of the IVGf- 1 culture, the follicle had grown noticeably (Fig. 3C). The growing follicle was removed from the gel and transferred to the collagen-coated membrane for IVGf-2 (Fig. 3D). After a further 8-day culture, the follicle formed an antral-like cavity indicated by an asterisk $\left(^{*}\right)$ in Fig. 3E. The follicle were mechanically removed from the membrane and transferred to the IVM culture. The matured eggs were fertilized by IVF and developed into blastocysts (Fig. 3F and G).

\section{Comparison of follicle and oocyte growth in groups $A, B$, and $C$}

Figure 4 shows the increase in follicle and oocyte diameters during a whole culture series (IVGf-1 and IVGf-2). The diameters of follicles and oocytes increased depending on their original sizes. The follicle diameter increased more during IVGf-2 than during IVGf- 1 in the three groups. Distribution of the diameters in the grown follicles was larger at day 17 (Fig. 4A). On the other hand, oocyte diameters increased in IVGf-1 culture rather than in IVGf-2 culture (Fig. 4B). The follicle diameters seemed to be determined by granulosa cell proliferation. At the end of the whole culture series, the differences in oocyte diameters were smaller than those of follicle diameters in each group. Physical oocyte growth mainly occurred in IVGf-1, and functional processes such as nuclear/cytoplasmic maturation occurred in IVGf-2. 
Table 2 Acquisition of competent oocytes after in vitro growth and maturation of primary/early secondary growing follicles.

\begin{tabular}{|c|c|c|c|c|c|}
\hline \multirow{4}{*}{$\begin{array}{l}\begin{array}{l}\text { Group } \\
\text { (follicle diameter) }\end{array} \\
\mathrm{A}(80-95 \mu \mathrm{m})\end{array}$} & \multirow{4}{*}{$\begin{array}{c}\begin{array}{c}\text { Follicles used } \\
\text { for culture }\end{array} \\
315\end{array}$} & \multicolumn{2}{|c|}{ No. of growing follicles after } & & \\
\hline & & $\begin{array}{l}\text { IVGf-1 } \\
\text { (at day } 9 \text { ) }\end{array}$ & $\begin{array}{l}\text { IVGf- } 1+\text { IVGf- } 2 \\
\text { (at day 17) }\end{array}$ & \multicolumn{2}{|c|}{$\begin{array}{l}\text { Nuclear status after } \\
\text { IVGf-1 + IVGf-2 + IVM }\end{array}$} \\
\hline & & $248(78.7 \%)^{*}$ & $194(61.6 \%)^{*}$ & MII & 95 (49.0\%) \\
\hline & & & & GVBD & $91(46.9 \%)$ \\
\hline & & & & GV & $8(4.1 \%)$ \\
\hline \multirow{3}{*}{$\mathrm{B}(70-80 \mu \mathrm{m})$} & 325 & $158(48.6 \%)^{\dagger}$ & $109(33.5 \%)^{\dagger}$ & MII & 64 (58.7\%) \\
\hline & & & & GVBD & $34(31.2 \%)$ \\
\hline & & & & GV & $11(10.1 \%)$ \\
\hline \multirow{3}{*}{$\mathrm{C}(60-70 \mu \mathrm{m})$} & 323 & $58(18.0 \%)^{\ddagger}$ & $32(9.9 \%)^{\neq}$ & MII & $16(50.0 \%)$ \\
\hline & & & & GVBD & $12(37.5 \%)$ \\
\hline & & & & GV & $4(12.5 \%)$ \\
\hline
\end{tabular}

Significant differences of follicle growth rate are compared with three groups $\left({ }^{*,+} P<0.001,{ }^{+, \ddagger} P<0.001\right)$. No significant differences of nuclear status are detected among three groups. IVGf, in vitro growth of follicles; IVM, in vitro maturation; GV, germinal vesicle; GVBD, germinal vesicle breakdown; MII, metaphase II.

\section{IVM and IVF of oocytes derived from two-step IVGf}

The growing follicles having an antral-like cavity at day 17 were transferred to the IVM medium to induce maturation. After $19 \mathrm{~h}$, most follicles released mucified cumulus-oocyte complexes (COCs) in the three groups. There were no significant differences in the nuclear maturation rates among them (Table 2). The terminal follicle size can be quite different among the three groups (groups $\mathrm{A}$ and $\mathrm{B}$ vs group $\mathrm{C}$ ) but this does not appear to have any significant bearing on the ultimate meiotic and developmental competence of the egg. When the mucified COCs that included oocytes with GVBD or MII stage were subjected to IVF, fertilization rates in groups $\mathrm{A}, \mathrm{B}$, and $\mathrm{C}$ were shown to be $39.2 \%$ (49/125), $51.0 \%(25 / 49)$, and $50.0 \%$ (7/14) respectively. The development rates of the zygotes to blastocysts in groups A, B, and C were $32.7 \%$ (16/49), 28.0\% (7/25), and $28.6 \%(2 / 7)$ respectively. There were no significant differences in fertilization rates and blastocyst development rates among the three groups.

\section{Live births from embryos derived from the two-step IVGf (IVGf-1 and IVGf-2), IVM, and IVF}

The two-cell-stage embryos obtained from the two-step IVGf, IVM, and IVF were vitrified to collect sufficient
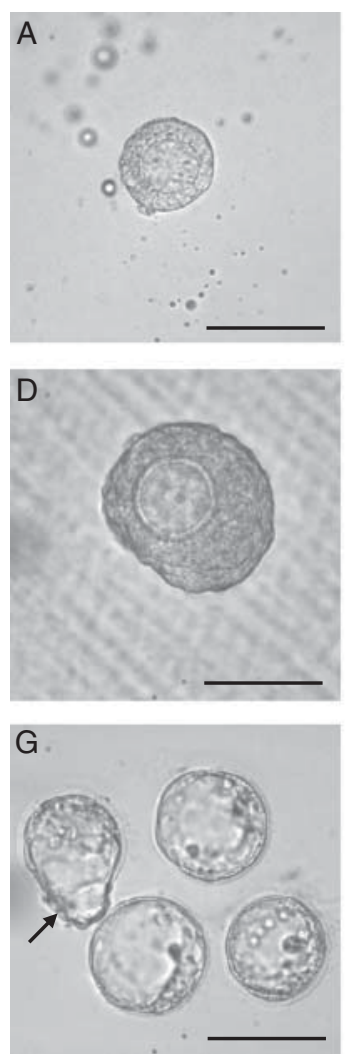
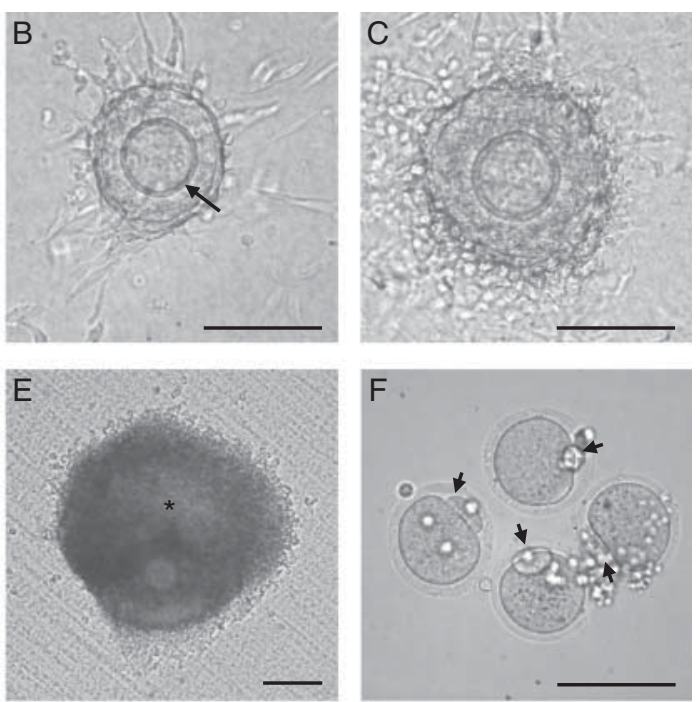

Figure 3 Morphological observation of follicle growth and embryo development in in vitro growth of follicles-1 (IVGf-1), in vitro growth of follicles-2 (IVGf-2), and in vitro maturation (IVM) followed by IVF. (A, B, and C) Follicle development from 6-day-old mouse ovaries during 9 days in IVGf-1. (A) At day 0 of the culture, an early secondary follicle with a centrally located oocyte embedded in collagen gel. (B) At day 5 of the culture, growing follicle with growing oocyte. Zona pellucida can be clearly seen (arrow). (C) At day 9 , further growing follicle in IVGf-1. Granulosa cells proliferated significantly compared with those shown in (B). (D and E) Subsequent follicle development in IVGf-2. (D) At day 9, the follicle was isolated from collagen gel and transferred to collagen-coated membrane. (E) At day 15, the follicle formed an antrallike cavity (asterisk). (F) Mature eggs derived from the series of two-step IVGf followed by IVM. They resumed meiosis and extruded first polar body (arrows). (G) Blastocysts after in vitro culture at $96 \mathrm{~h}$ from IVF. One is under hatching from zona pellucida (arrow). Scale bar $=100 \mu \mathrm{m}$. 

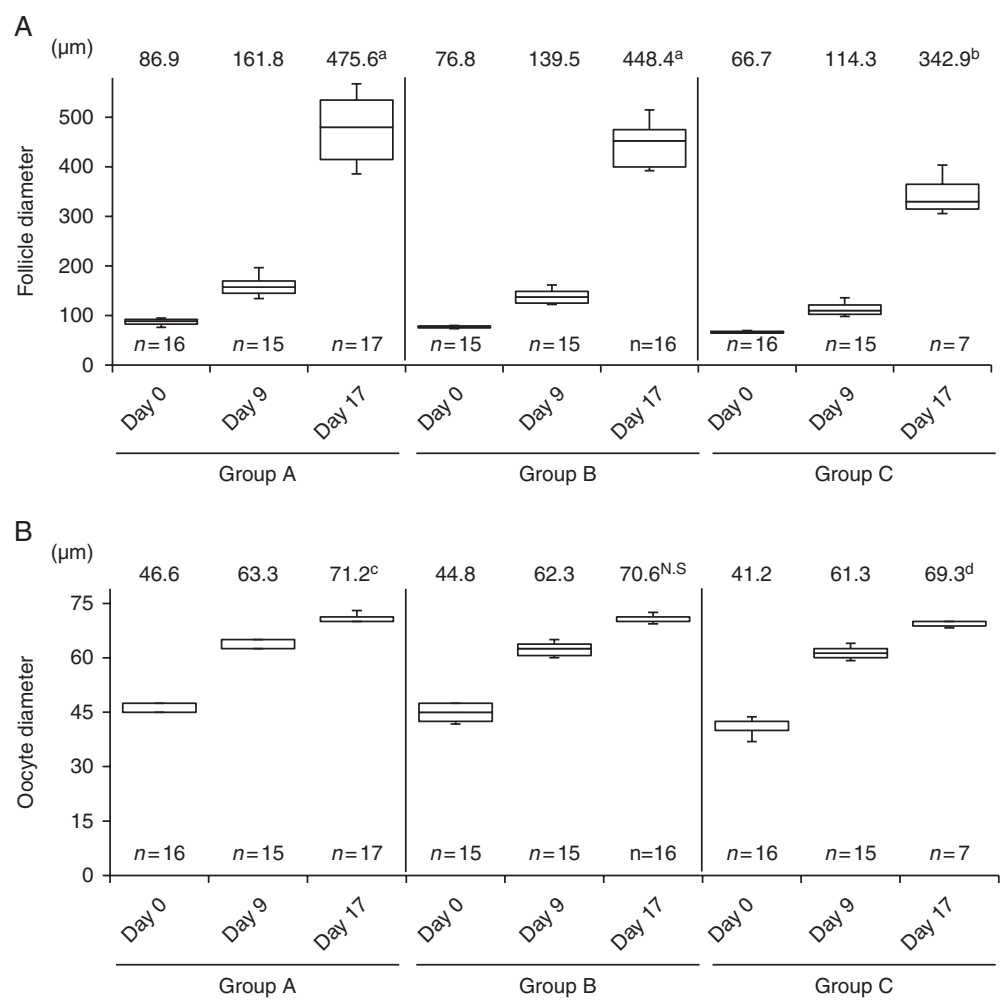

\begin{abstract}
Figure 4 Increase in follicle and oocyte diameters in the series of two-step culture system. In vitro growth culture started at day 0 to day 9 (IVGf-1) and subsequent culture was performed from day 10 to day 17 (IVGf-2). (A) Follicle diameter. (B) Oocyte diameter. Box-and-whiskers plot demonstrating association between diameter and days of culture. Box contains values between 25th and 75th percentiles of diameter (central line, median). Vertical lines represent 10th and 90th percentiles. Numbers above the box plot indicate mean diameters $(\mu \mathrm{m})$, and different letters beside the mean diameters indicate significant differences among the different groups at day 17 of the culture $(P<0.05)$.
\end{abstract}

numbers for embryo transfer. One hundred and thirtyone embryos at the two-cell stage from three-run experiments were cryopreserved. After warming, $96.2 \%(126 / 131)$ survived. Fifty-three embryos that developed into the four-cell stage after overnight culture were selected and combined from the three groups for transfer to four pseudopregnant mice; therefore, which follicle group the live offspring were derived from could not be distinguished. The remaining embryos were cultured in modified KSOM for $60 \mathrm{~h}$ to examine developmental competence to blastocyst in vitro. Three mice became pregnant and two offspring were born by Cesarean section from two pregnant mice (Fig. 5 and Table 3). Their body/placenta appearance and weights were normal, and abnormality was not observed at birth. However, in the uteri, there were several dead embryos at mid stage of gestation. Also there was a fetus that was considered to have been alive to just before full term in the uterus of the other pregnant mouse. The two live offspring were females and started breathing spontaneously. They grew up and matured sexually to show normal reproductive ability.

\section{Discussion}

Developing a culture system of small ovarian follicles is important, because the ability to culture oocytes from the relatively abundant primary stage would provide a research tool to study the complex process of follicle development. A mouse model is useful for this purpose. Several culture methods were applied to preantral mouse follicles with diameters of 110-140 $\mu$ m (Cortvrindt \& Smitz 1998, Kreeger et al. 2005, Wang et al. 2011). However, these methods are still insufficient for earlier preantral stages. The study presented here was designed to establish an IVG system to induce the growth of earlier stage follicles such as primary/early secondary stages with diameters $<95 \mu \mathrm{m}$. We assumed that physical maintenance of follicle structure was important to give the granulosa cells the opportunity to increase in number of layers. To verify this assumption, we used collagen gel culture (IVGf-1) before a collagen-coated membrane method (IVGf-2) for growth induction of primary/early secondary follicles. By embedding such small follicles in collagen gel, suppression of rapid migration of follicular cell occurred and three-dimensional follicle structure was maintained. It suggests that the two-step culture method for primary/early secondary follicles is very successful if organ culture is not applied to the culture system.

A number of researchers have reported that the covering of oocytes with granulosa cells and the keeping of junctional contacts among adjacent granulosa cells are essential for normal follicle growth. Autocrine/ paracrine systems in the follicle seemed not to be retained in plain coculture systems of oocytes and granulosa cells. Oocytes in primary/early secondary follicles did not form functional mature oocytes having fertilizing ability in the coculture system, either (Eppig 1979, Eppig \& Schroeder 1989). Therefore, maintaining normal follicle structure was considered to be crucial for successful follicle growth in culture. 

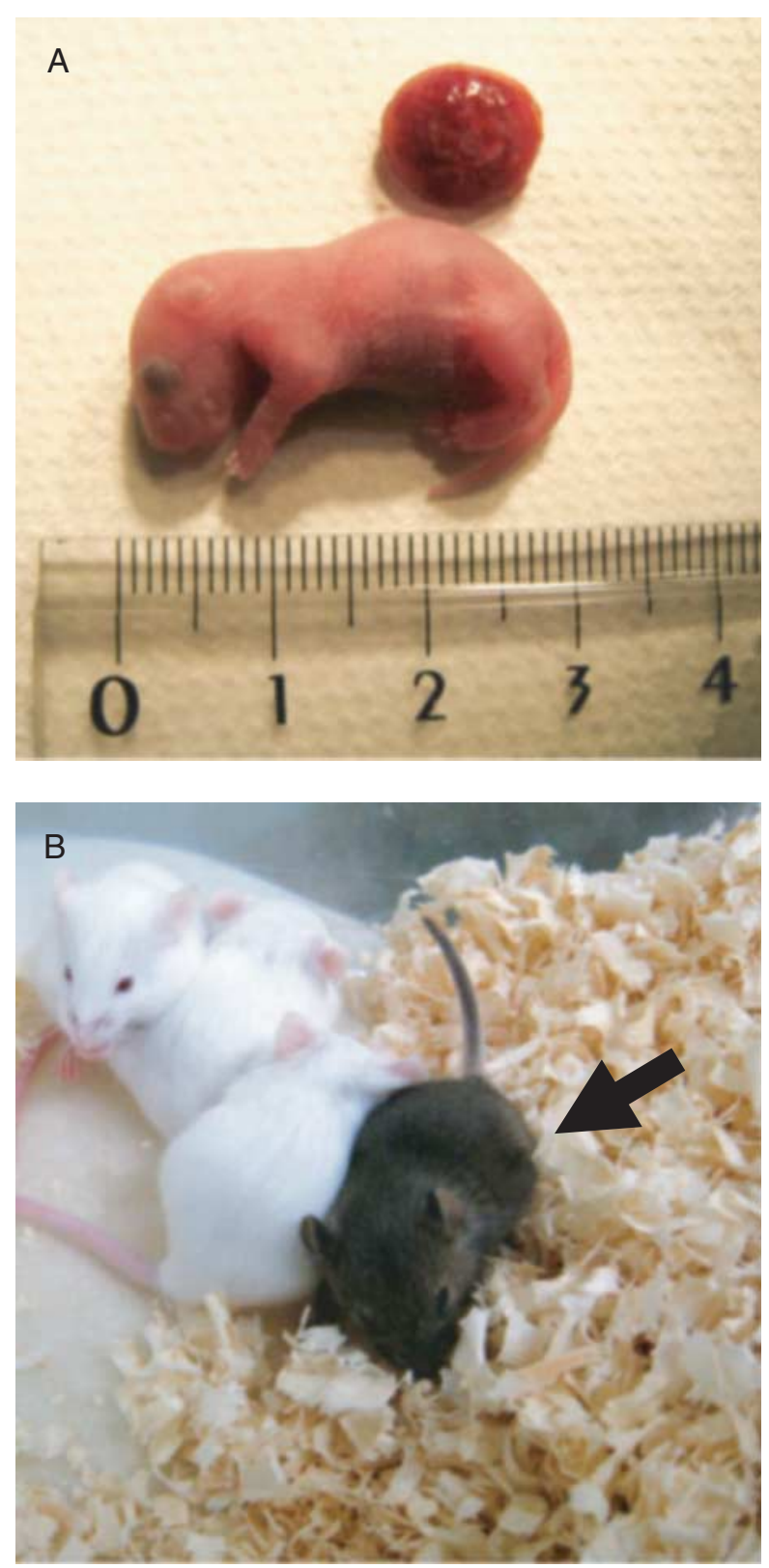

Figure 5 Live offspring derived from primary/early secondary follicles cultured by the series of two-step in vitro growth of follicles (IVGf), in vitro maturation (IVM), IVF followed by embryo transfer.

(A) Live offspring delivered by Cesarean section and its placenta.

(B) The growing mouse at 15 days after birth (arrow).

In this study, we used collagen gel, an extracellular matrix, for the culture substratum. This matrix probably gives growth signals to the follicles as well as mechanical stability. Actually, collagen has been reported to regulate ovarian functions (Matsumura et al. 2009). Under IVGf-1 culture, we succeeded in the induction of small follicle growth having diameters of $60-70 \mu \mathrm{m}$ designated group C. These oocytes reached mature stages after subsequent culture of IVGf-2 and IVM. The oocytes were fertilized by IVF and the derived zygotes developed into blastocysts.
Transfer of growing follicles to IVGf-2 from IVGf-1 is essential for sufficient growth of the follicles. If IVGf-1 continues for 12 days, the follicles degenerate in the collagen gels (data not shown). Similar observations have been reported in collagen gel culture (Torrance et al. 1989). The reason why the follicles stop growing in the continuous collagen gel culture is not clear, but it is possible that too much follicular cell proliferation results in a shortage of necessary nutritional and/or growth factors. As another explanation, toxic metabolites may be released from follicular cells and their local densities may easily increase due to the viscosity of the gel. Recently, a novel hypothesis has been proposed (Woodruff \& Shea 2011); follicle growth is dependent on the physical environment of the ovary in addition to well-established hormone control. During follicle development, early growing follicles move from the cortex, a collagen-rich zone, to perimedullar region of ovary and move again to the ovarian surface prior to ovulation. This study suggests that the change in the physical environment from rigid gel (IVGf-1) to a less rigid liquid culture (IVGf-2) may recreate the situation of follicle growth in the ovary. Large mammalian species such as human follicles may have diameters $>20 \mathrm{~mm}$ at final stage but they are still small at primary/early secondary stages. Therefore, IVGf-1 using a small amount of the gel followed by IVGf-2 using a large amount of liquid medium will support the follicle growth of large mammalian species.

Gas conditions in culture are also important. When gas conditions for IVGf-1 were checked, a condition of $5 \%$ $\mathrm{CO}_{2}, 5 \% \mathrm{O}_{2}$, and $90 \% \mathrm{~N}_{2}$ showed a higher follicle growth than that of $5 \% \mathrm{CO}_{2}$ in air. In the latter condition, the follicles degenerated with higher rates. A few follicles from group A grew, but the diameters were smaller compared with those observed in the former condition. The follicles from groups $\mathrm{B}$ and $\mathrm{C}$ were denuded and degenerated within $24 \mathrm{~h}$ in the latter condition. This suggests that $5 \% \mathrm{O}_{2}$ conditions are suitable for primary/early secondary follicles during early growth phase. It is known that angiogenesis in physiological conditions occurs markedly during progress of ovarian development (Fraser 2006). Several groups reported that modulating oxygen concentration was effective in follicle culture (Heise et al. 2009, Hirao et al. 2012).

Table 3 Live offspring from early growing follicles after in vitro culture followed by ET.

\begin{tabular}{lccc}
\hline Recipient ID & $\begin{array}{c}\text { No. of embryos } \\
\text { transferred }\end{array}$ & No. of IUFD & No. of live offspring \\
\hline 1 & 11 & 2 & $\begin{array}{c}1 \text { (female, bw: } 1.990, \\
\text { pw: } 0.232)\end{array}$ \\
2 & 9 & 2 & $\begin{array}{c}\text { (female, bw: } 2.044 \\
\text { pw: } 0.243)\end{array}$ \\
\hline
\end{tabular}

IUFD, intrauterine fetal death; bw, body weight (g); pw, placenta weight (g). Embryos were developed from 6-day-old mouse ovarian follicles after two-step IVGf, IVM, and IVF. 
Taken together, $\mathrm{O}_{2}$ supply seems to be necessary for the later stages of folliculogenesis. In our own findings, the change of gas conditions from IVGf-1 to IVGf-2 was also important to imitate physiological conditions to achieve high success rates of follicle growth.

Although we succeeded in inducing primary follicles to develop into fertilizable mature eggs, the growth rate of group $C$ was lower than that of the other groups during IVGf-1. Less-developed granulosa cells of primary/early secondary follicles very often adhered to the collagencoated membrane or even the collagen gel, probably due to the granulosa cells' nature and/or immature communications between oocyte and granulosa cells such as poor gap junction formation.

When comparing the fertilization rates and blastocystachieving rates from two pronuclei-formed zygotes, there were no significant differences among the three groups classified by follicular diameters. However, the fertilization and blastocyst-achieving rates from any group were lower than those of in vivo grown oocytes (data is not shown). In the study presented here, we demonstrated the production of two live offspring (3.7\%) from transferred embryos derived from oocytes grown in vitro from primary/early secondary follicles without organ culture. The success rate of live births was still low similar to the previous studies (Eppig \& Schroeder 1989, Spears et al. 1994, Eppig \& O'Brien 1996, O'Brien et al. 2003, Xu et al. 2006). Further efforts are needed to improve culture conditions.

Lenie reported a multistep culture system that changed concentrations of FCS in the medium in order to develop isolated ovarian follicles smaller than $100 \mu \mathrm{m}$ but they did not examine developmental competence to birth (Lenie et al. 2004). Currently, different culture methods have been reported that support the growth and survival of isolated primary/early secondary follicles. These methods have demonstrated that co-culturing earlystage follicles encapsulated in alginate with mouse embryonic fibroblasts or ovarian stromal cells improves follicle growth and survival (Tingen et al. 2011, Tagler et al. 2012). Furthermore, group-cultured primary follicles have been reported to survive and effectively grow when compared with those in individual culture (Hornick et al. 2013). It seems that follicles themselves can exert a beneficial coculture effect. These results suggest that early-stage follicles may require unidentified factors responsible for promoting their growth and survival. We should also develop components of the culture medium as well as culture devices.

Recently, the activation mechanism of dormant primordial follicles has been reported in mice (Reddy et al. 2008). However, the molecular mechanisms underlying follicle selection and development from primary stage to preovulatory stage remain poorly understood. It is important that follicle culture methods, not culture of ovarian tissues, can provide the opportunity to study regulatory mechanisms of folliculogenesis.
We would be able to study molecular mechanisms of follicular development, selection, and atresia using uniform follicles that were categorized by oocyte diameters and the number of granulosa cell layers, as opposed to a mixture of follicles at various developmental stages in ovarian tissue. The somatic component of stromal tissue associated with intraovarian regulatory process is not known. To clarify this, we can design experiments without systemic influences, as follicles in ovarian tissue would be inevitably affected by around environment.

It has been recognized that the efficiency of assisted reproductive technology could be enhanced by using oocytes from immature follicles for IVG and subsequent IVM. However, adjustment of optimal culture conditions is difficult for follicles at different stages because human follicles isolated from ovarian tissue vary in number and growth stage. Consequently, the technologies of IVG from various stage follicles would offer new applications for fertility preservation. This culture system provides the first encouraging step toward achieving full IVG of human small follicles, although we should make further effort to develop a more effective culture system. Also, we demonstrated that vitrification can be applicable to adequate embryo transfer. A combination of IVGf and vitrification may become a feasible method for future infertility treatment, because the vitrification technique has already been widely applied for clinical use (Gosden 2011, Herrero et al. 2011).

In conclusion, this study presents a two-step IVGf system for primary/early secondary follicles comprising small oocytes (diameters of $\sim 45 \mu \mathrm{m}$ ), with one to three granulosa cell layers and less-developed theca cells. The oocytes in the grown follicles from this system resumed meiosis and fertilized with high success rates. The presumed embryos developed to live offspring by embryo transfer after vitrification and warming at four-cell stage. To our knowledge, this is the first report of offspring born after culture of individual follicles (diameters of $<100 \mu \mathrm{m}$ ) followed by IVGf, IVM, and IVF-ET.

\section{Declaration of interest}

The authors declare that there is no conflict of interest that could be perceived as prejudicing the impartiality of the research reported.

\section{Funding}

This work was supported in part by a Grant-in-Aid for Scientific Research (C) (no. 23592427) from the Japan Society for the Promotion of Science.

\section{References}

Andersen CY, Rosendahl M, Byskov AG, Loft A, Ottosen C, Dueholm M, Schmidt KL, Andersen AN \& Ernst E 2008 Two successful pregnancies following autotransplantation of frozen/thawed ovarian tissue. Human Reproduction 23 2266-2272. (doi:10.1093/humrep/den244) 
Anderson RA, Wallace WH \& Baird DT 2008 Ovarian cryopreservation for fertility preservation: indications and outcomes. Reproduction 136 681-689. (doi:10.1530/REP-08-0097)

Berkholtz CB, Lai BE, Woodruff TK \& Shea LD 2006 Distribution of extracellular matrix proteins type I collagen, type IV collagen, fibronectin, and laminin in mouse folliculogenesis. Histochemistry and Cell Biology 126 583-592. (doi:10.1007/s00418-006-0194-1)

Carroll J, Whittingham DG \& Wood MJ 1991 Effect of gonadotrophin environment on growth and development of isolated mouse primary ovarian follicles. Journal of Reproduction and Fertility 93 71-79. (doi:10.1530/jrf.0.0930071)

Cortvrindt R \& Smitz J 1998 Early preantral mouse follicle in vitro maturation: oocyte growth, meiotic maturation and granulosa-cell proliferation. Theriogenology 49 845-859. (doi:10.1016/S0093691X(98)00034-X)

Cortvrindt R, Smitz J \& Van Steirteghem AC 1996 In-vitro maturation, fertilization and embryo development of immature oocytes from early preantral follicles from prepuberal mice in a simplified culture system. Human Reproduction 11 2656-2666. (doi:10.1093/oxfordjournals. humrep.a019188)

Demeestere I, Simon P, Emiliani S, Delbaere A \& Englert Y 2007 Fertility preservation: successful transplantation of cryopreserved ovarian tissue in a young patient previously treated for Hodgkin's disease. Oncologist 12 1437-1442. (doi:10.1634/theoncologist.12-12-1437)

Donnez J, Dolmans MM, Demylle D, Jadoul P, Pirard C, Squifflet J, Martinez-Madrid B \& van Langendonckt A 2004 Livebirth after orthotopic transplantation of cryopreserved ovarian tissue. Lancet $\mathbf{3 6 4}$ 1405-1410. (doi:10.1016/S0140-6736(04)17222-X)

Eppig JJ 1979 A comparison between oocyte growth in coculture with granulosa cells and oocytes with granulosa cell-oocyte junctional contact maintained in vitro. Journal of Experimental Zoology 209 345-353. (doi:10.1002/jez.1402090216)

Eppig JJ \& O'Brien MJ 1996 Development in vitro of mouse oocytes from primordial follicles. Biology of Reproduction 54 197-207. (doi:10.1095/ biolreprod54.1.197)

Eppig JJ \& Schroeder AC 1989 Capacity of mouse oocytes from preantral follicles to undergo embryogenesis and development to live young after growth, maturation, and fertilization in vitro. Biology of Reproduction 41 268-276. (doi:10.1095/biolreprod41.2.268)

Fraser HM 2006 Regulation of the ovarian follicular vasculature. Reproductive Biology and Endocrinology 4 18. (doi:10.1186/14777827-4-18)

Gosden R 2011 Cryopreservation: a cold look at technology for fertility preservation. Fertility and Sterility 96 264-268. (doi:10.1016/j.fertnstert. 2011.06.029)

Hasegawa A, Mochida N, Ogasawara T \& Koyama K 2006 Pup birth from mouse oocytes in preantral follicles derived from vitrified and warmed ovaries followed by in vitro growth, in vitro maturation, and in vitro fertilization. Fertility and Sterility 86 1182-1192. (doi:10.1016/j. fertnstert.2005.12.082)

Heise MK, Koepsel R, McGee EA \& Russell AJ 2009 Dynamic oxygen enhances oocyte maturation in long-term follicle culture. Tissue Engineering Part C Methods 15 323-332. (doi:10.1089/ten.tec.2007. 0418)

Herrero L, Martinez M \& Garcia-Velasco JA 2011 Current status of human oocyte and embryo cryopreservation. Current Opinion in Obstetrics \& Gynecology 23 245-250. (doi:10.1097/GCO.0b013e32834874e2)

Hirao Y \& Miyano T 2008 In vitro growth of mouse oocytes: oocyte size at the beginning of culture influences the appropriate length of culture period. Journal of Mammalian Ova Research 25 56-62. (doi:10.1274/ jmor.25.56)

Hirao Y, Itoh T, Shimizu M, Iga K, Aoyagi K, Kobayashi M, Kacchi M, Hoshi H \& Takenouchi N 2004 In vitro growth and development of bovine oocyte-granulosa cell complexes on the flat substratum: effects of high polyvinylpyrrolidone concentration in culture medium. Biology of Reproduction 70 83-91. (doi:10.1095/biolreprod.103.021238)

Hirao Y, Shimizu M, Iga K \& Takenouchi N 2012 Optimization of oxygen concentration for growing bovine oocytes in vitro: constant low and high oxygen concentrations compromise the yield of fully grown oocytes. Journal of Reproduction and Development 58 204-211. (doi:10.1262/ jrd.11-132M)
Hornick JE, Duncan FE, Shea LD \& Woodruff TK 2013 Multiple follicle culture supports primary follicle growth through paracrine-acting signals. Reproduction 145 19-32. (doi:10.1530/REP-12-0233)

Hwang DH, Kee SH, Kim K, Cheong KS, Yoo YB \& Lee BL 2000 Role of reconstituted basement membrane in human granulosa cell culture. Endocrine Journal 47 177-183. (doi:10.1507/endocrj.47.177)

Jin SY, Lei L, Shikanov A, Shea LD \& Woodruff TK 2010 A novel two-step strategy for in vitro culture of early-stage ovarian follicles in the mouse. Fertility and Sterility 93 2633-2639. (doi:10.1016/j.fertnstert.2009.10.027)

Kreeger PK, Fernandes NN, Woodruff TK \& Shea LD 2005 Regulation of mouse follicle development by follicle-stimulating hormone in a three-dimensional in vitro culture system is dependent on follicle stage and dose. Biology of Reproduction 73 942-950. (doi:10.1095/biolreprod.105.042390)

Kreeger PK, Deck JW, Woodruff TK \& Shea LD 2006 The in vitro regulation of ovarian follicle development using alginate-extracellular matrix gels. Biomaterials 27 714-723. (doi:10.1016/j.biomaterials.2005.06.016)

Lenie S, Cortvrindt R, Adriaenssens T \& Smitz J 2004 A reproducible twostep culture system for isolated primary mouse ovarian follicles as single functional units. Biology of Reproduction 71 1730-1738. (doi:10.1095/ biolreprod.104.028415)

Lutchman Singh K, Davies M \& Chatterjee R 2005 Fertility in female cancer survivors: pathophysiology, preservation and the role of ovarian reserve testing. Human Reproduction Update 11 69-89. (doi:10.1093/humupd/ dmh052)

Matsumura H, Kano K, Marin de Evsikova C, Young JA, Nishina PM, Naggert JK \& Naito K 2009 Transcriptome analysis reveals an unexpected role of a collagen tyrosine kinase receptor gene, Ddr2, as a regulator of ovarian function. Physiological Genomics 39 120-129. (doi:10.1152/physiolgenomics.00073.2009)

Meirow D \& Nugent D 2001 The effects of radiotherapy and chemotherapy on female reproduction. Human Reproduction Update 7 535-543. (doi:10.1093/humupd/7.6.535)

Meirow D, Levron J, Eldar-Geva T, Hardan I, Fridman E, Zalel Y, Schiff E \& Dor J 2005 Pregnancy after transplantation of cryopreserved ovarian tissue in a patient with ovarian failure after chemotherapy. New England Journal of Medicine 353 318-321. (doi:10.1056/NEJMc055237)

Meirow D, Hardan I, Dor J, Fridman E, Elizur S, Ra'anani H, Slyusarevsky E, Amariglio N, Schiff E, Rechavi G et al. 2008 Searching for evidence of disease and malignant cell contamination in ovarian tissue stored from hematologic cancer patients. Human Reproduction 23 1007-1013. (doi:10.1093/humrep/den055)

Nayudu PL \& Osborn SM 1992 Factors influencing the rate of preantral and antral growth of mouse ovarian follicles in vitro. Journal of Reproduction and Fertility 95 349-362. (doi:10.1530/jrf.0.0950349)

O'Brien MJ, Pendola JK \& Eppig JJ 2003 A revised protocol for in vitro development of mouse oocytes from primordial follicles dramatically improves their developmental competence. Biology of Reproduction $\mathbf{6 8}$ 1682-1686. (doi:10.1095/biolreprod.102.013029)

Pangas SA, Saudye H, Shea LD \& Woodruff TK 2003 Novel approach for the three-dimensional culture of granulosa cell-oocyte complexes. Tissue Engineering 9 1013-1021. (doi:10.1089/107632703322495655)

Pedersen T \& Peters H 1968 Proposal for a classification of oocytes and follicles in the mouse ovary. Journal of Reproduction and Fertility 17 555-557. (doi:10.1530/jrf.0.0170555)

Reddy P, Liu L, Adhikari D, Jagarlamudi K, Rajareddy S, Shen Y, Du C, Tang W, Hamalainen T, Peng SL et al. 2008 Oocyte-specific deletion of Pten causes premature activation of the primordial follicle pool. Science 319 611-613. (doi:10.1126/science.1152257)

Scott JE, Carlsson IB, Bavister BD \& Hovatta O 2004 Human ovarian tissue cultures: extracellular matrix composition, coating density and tissue dimensions. Reproductive BioMedicine Online 9 287-293. (doi:10.1016/S1472-6483(10)62143-8)

Shaw JM, Bowles J, Koopman P, Wood EC \& Trounson AO 1996 Fresh and cryopreserved ovarian tissue samples from donors with lymphoma transmit the cancer to graft recipients. Human Reproduction 11 1668-1673. (doi:10.1093/oxfordjournals.humrep.a019467)

Smitz JE \& Cortvrindt RG 2002 The earliest stages of folliculogenesis in vitro. Reproduction 123 185-202. (doi:10.1530/rep.0.1230185)

Spears N, Boland NI, Murray AA \& Gosden RG 1994 Mouse oocytes derived from in vitro grown primary ovarian follicles are fertile. Human Reproduction 9 527-532. 
Tagler D, Tu T, Smith RM, Anderson NR, Tingen CM, Woodruff TK \& Shea LD 2012 Embryonic fibroblasts enable the culture of primary ovarian follicles within alginate hydrogels. Tissue Engineering, Part A 18 1229-1238. (doi:10.1089/ten.tea.2011.0418)

Telfer EE, McLaughlin M, Ding C \& Thong KJ 2008 A two-step serum-free culture system supports development of human oocytes from primordial follicles in the presence of activin. Human Reproduction 23 1151-1158. (doi:10.1093/humrep/den070)

Tingen CM, Kiesewetter SE, Jozefik J, Thomas C, Tagler D, Shea L \& Woodruff TK 2011 A macrophage and theca cell-enriched stromal cell population influences growth and survival of immature murine follicles in vitro. Reproduction 141 809-820. (doi:10.1530/REP-10-0483)

Torrance C, Telfer E \& Gosden RG 1989 Quantitative study of the development of isolated mouse pre-antral follicles in collagen gel culture. Journal of Reproduction and Fertility 87 367-374. (doi:10.1530/ jrf.0.0870367)

Wang X, Catt S, Pangestu M \& Temple-Smith P 2011 Successful in vitro culture of pre-antral follicles derived from vitrified murine ovarian tissue: oocyte maturation, fertilization, and live births. Reproduction 141 183-191. (doi:10.1530/REP-10-0383)

Wicha MS, Liotta LA, Garbisa S \& Kidwell WR 1979 Basement membrane collagen requirements for attachment and growth of mammary epithelium. Experimental Cell Research 124 181-190. (doi:10.1016/ 0014-4827(79)90268-4)

Woodruff TK \& Shea LD 2011 A new hypothesis regarding ovarian follicle development: ovarian rigidity as a regulator of selection and health. Journal of Assisted Reproduction and Genetics 28 3-6. (doi:10.1007/ s10815-010-9478-4)
Wycherley G, Downey D, Kane MT \& Hynes AC 2004 A novel follicle culture system markedly increases follicle volume, cell number and oestradiol secretion. Reproduction 127 669-677. (doi:10.1530/rep.1. 00040)

Xu M, Kreeger PK, Shea LD \& Woodruff TK 2006 Tissue-engineered follicles produce live, fertile offspring. Tissue Engineering 12 2739-2746. (doi:10.1089/ten.2006.12.2739)

Xu M, Barrett SL, West-Farrell E, Kondapalli LA, Kiesewetter SE, Shea LD \& Woodruff TK 2009a In vitro grown human ovarian follicles from cancer patients support oocyte growth. Human Reproduction 24 2531-2540. (doi:10.1093/humrep/dep228)

Xu M, West-Farrell ER, Stouffer RL, Shea LD, Woodruff TK \& Zelinski MB $2009 b$ Encapsulated three-dimensional culture supports development of nonhuman primate secondary follicles. Biology of Reproduction $\mathbf{8 1}$ 587-594. (doi:10.1095/biolreprod.108.074732)

Yang J \& Nandi S 1983 Growth of cultured cells using collagen as substrate. International Review of Cytology 81 249-286.

Yang J, Guzman R, Richards J \& Nandi S 1980 Primary culture of mouse mammary tumor epithelial cells embedded in collagen gels. In Vitro $\mathbf{1 6}$ 502-506. (doi:10.1007/BF02626463)

Received 19 January 2013

First decision 19 February 2013

Revised manuscript received 12 April 2013

Accepted 22 April 2013 